\title{
Features of electromagnetic wave propagation in two- and three-layer cylindrical dielectric waveguides
}

\author{
Alexander G. Shein ${ }^{1, *}$ and Tatiana $S$. Kharlanova ${ }^{1}$ \\ ${ }^{1}$ Volgograd State Technical University, V. I. Lenin Avenue, 28, Volgograd, 400005, Russia
}

\begin{abstract}
Various characteristics of electromagnetic waves propagating in two- and three-layer open-dielectric waveguide structures of cylindrical shape are studied. Parameters of wave components in separate areas of the waveguide are considered. Electrodynamic characteristics and frequency dependencies for several values of layer dielectric permittivity obtained during the study are analyzed. The corresponding graphs of dependencies are presented. The dependencies of the power flux density of transverse magnetic (TM) waves in two- and three-layer waveguide structures in the absence of field dependence on azimuthal angle are considered.
\end{abstract}

\section{Introduction}

Round waveguides are widely used in the development of SHF and EHF devices. Of great interest is the study of the structure and characteristics of electromagnetic waves propagating in multilayer dielectric waveguides at different values of the relative dielectric permittivity of individual layers. Of equal interest are the conditions for the appearance of different types of waves with different velocities and critical frequencies at certain frequencies. Identifying certain parameters of the waveguide (radii of the layer cross-section, relative dielectric permittivity, relations between them for neighboring layers) in order to obtain the maximum concentration of the flow power in a certain layer is also very relevant.

Cylindrical waveguides are a suitable example of a guiding structure for studying electrodynamic characteristics [3]. The theory of multilayer metal-dielectric and dielectric waveguides, peculiarities of wave propagation in two- and three-layer waveguides are considered in the literature [3-7]. Of interest are the peculiarities of the behavior of electromagnetic waves in multilayer dielectric waveguides, in which the dielectric permittivity of the layers is different, that is, the ratio between them varies.

To find the structure of the electromagnetic field and wave parameters in the waveguide it is necessary to obtain a dispersion equation, for which the system of Maxwell equations with the corresponding boundary conditions is solved. In order for the boundary conditions are satisfied at all points on the waveguide surface at any given time, the propagation constant (wave number) $\beta$ must be the same inside and outside the waveguide [6-11].

\footnotetext{
* Corresponding author: professor39@mail.ru
} 


\section{The subject}

There is an open cylindrical waveguide consisting of several layers of dielectric: each of the layers has a cross section radius $r_{n}$, relative dielectric permittivity $\varepsilon_{n}$, relative magnetic permittivity $\mu_{n}$. The surrounding space is considered as a dielectric layer of a waveguide with infinitely extended walls with dielectric permittivity $\varepsilon_{n+1}$ and magnetic permittivity $\mu_{n+1}$ (Fig. 1)

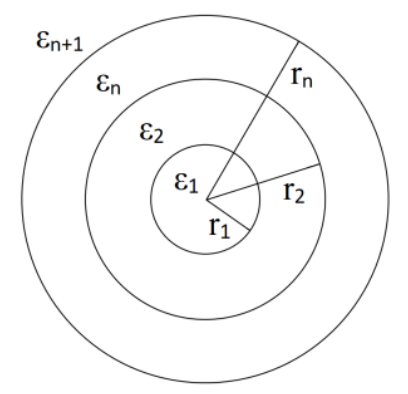

Fig. 1. General view of the cylindrical multilayer dielectric waveguide.

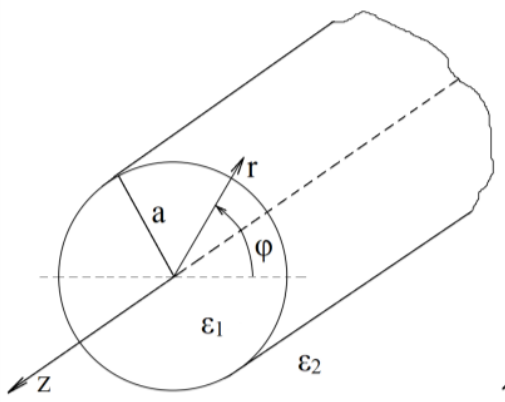

a)

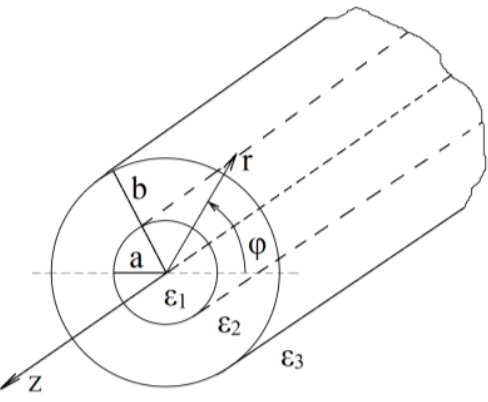

b)

Fig. 2. Two- (a) and three-layer (b) cylindrical waveguide structure.

It is assumed that the dielectric is homogeneous and isotropic in each of the layers of the waveguide, and losses in the process of electromagnetic wave propagation are not taken into account. The cases for different ratios between the relative dielectric permittivity of the layers, as well as for different radii of the waveguide cross-section during the propagation of symmetrical and asymmetrical waves are considered.

Calculations for sufficiently small values of radii of cross-section of the waveguide layers $\mathrm{r} \sim 10^{-3} \mathrm{~m}$ at the frequencies of electromagnetic waves of the EHF range were carried out. The cases when the value of dielectric permittivity was the highest in the inner layer were considered. In the course of calculations, the cases of a two-layer waveguide system (Fig.2, a) at a ratio of dielectric permittivities $\varepsilon_{1}>\varepsilon_{2}$ and a three-layer waveguide system (Fig.3, b) at a ratio of $\varepsilon_{1}>\varepsilon_{2}>\varepsilon_{3}, \varepsilon_{2}>\varepsilon_{1}>\varepsilon_{3}$ were analyzed.

\section{Dispersion relations}

\subsection{Expressions for electromagnetic wave components in a two-layer wave- guide structure. Case of $\varepsilon_{1}>\varepsilon_{2}, r_{1}=a, r_{2} \rightarrow \infty$ (Fig. 2, a). Dispersion relation}

In general, the expression for transverse wave numbers has the form: 


$$
k_{n}=\sqrt{\beta^{2}-\omega^{2} \varepsilon_{0} \mu_{0} \varepsilon_{n} \mu_{n}}
$$

For the inner layer $\left(\varepsilon_{1}, \mu_{1}\right)$ :

$$
\left.\begin{array}{c}
H_{z 1}^{m}=A_{1} J_{m}\left(k_{1} r\right) e^{ \pm i m \varphi} \\
E_{z 1}^{e}=B_{1} J_{m}\left(k_{1} r\right) e^{ \pm i m \varphi} \\
H_{\varphi 1}= \pm \frac{\beta m}{r k_{1}^{2}} A_{1} J_{m}\left(k_{1} r\right) e^{ \pm i m \varphi}+\frac{i \omega \varepsilon_{0} \varepsilon_{1}}{k_{1}} B_{1} J_{m}^{\prime}\left(k_{1} r\right) e^{ \pm i m \varphi} \\
E_{\varphi 1}= \pm \frac{\beta m}{r k_{1}^{2}} B_{1} J_{m}\left(k_{1} r\right) e^{ \pm i m \varphi}+\frac{i \omega \mu_{0} \mu_{1}}{k_{1}} A_{1} J_{m}^{\prime}\left(k_{1} r\right) e^{ \pm i m \varphi}
\end{array}\right\}
$$

For the outer layer $\left(\varepsilon_{2}, \mu_{2}\right)$ :

$$
\left.\begin{array}{c}
H_{z 2}^{m}=A_{2} K_{m}\left(k_{2} r\right) e^{ \pm i m \varphi} \\
E_{z 2}^{e}=B_{2} K_{m}\left(k_{2} r\right) e^{ \pm i m \varphi} \\
H_{\varphi 2}= \pm \frac{\beta m}{r k_{2}^{2}} A_{2} K_{m}\left(k_{2} r\right) e^{ \pm i m \varphi}+\frac{i \omega \varepsilon_{0} \varepsilon_{2}}{k_{2}} B_{2} K_{m}^{\prime}\left(k_{2} r\right) e^{ \pm i m \varphi} \\
E_{\varphi 3}= \pm \frac{\beta m}{r k_{2}^{2}} B_{2} K_{m}\left(k_{2} r\right) e^{ \pm i m \varphi}+\frac{i \omega \mu_{0} \mu_{2}}{k_{2}} A_{2} K_{m}^{\prime}\left(k_{2} r\right) e^{ \pm i m \varphi}
\end{array}\right\}
$$

Using the continuity boundary conditions for longitudinal and transverse components of the electromagnetic field at the layer interface $\left(r=a: E_{\varphi 1}(a)=E_{\varphi 2}(a), H_{\varphi 1}(a)=H_{\varphi 2}(a), E_{z 1}(a)=E_{z 2}(a), H_{z 1}(a)=H_{z 2}(a)\right)$ it is possible to obtain a dispersion relation for a two-layer waveguide structure [7-11]:

$$
(\beta m)^{2}\left(\frac{1}{k_{1}^{2}}+\frac{1}{k_{2}^{2}}\right)^{2}=(\omega a)^{2}\left[\frac{\mu_{0} \mu_{1}}{k_{1}} \frac{J_{m}^{\prime}\left(k_{1} a\right)}{J_{m}\left(k_{1} a\right)}-\frac{\mu_{0} \mu_{2}}{k_{2}} \frac{K_{m}^{\prime}\left(k_{2} a\right)}{K_{m}\left(k_{2} a\right)}\right]\left[\frac{\varepsilon_{0} \varepsilon_{1}}{k_{1}} \frac{J_{m}^{\prime}\left(k_{1} a\right)}{J_{m}\left(k_{1} a\right)}-\frac{\varepsilon_{0} \varepsilon_{2}}{k_{2}} \frac{K_{m}^{\prime}\left(k_{2} a\right)}{K_{m}\left(k_{2} a\right)}\right]
$$

Expression for interrelation between transverse and longitudinal wave numbers:

$$
\left(k_{1} a\right)^{2}+\left(k_{2} a\right)^{2}=\left(\mu_{1} \varepsilon_{1}-\mu_{2} \varepsilon_{2}\right) \frac{\omega^{2}}{c^{2}} a^{2}
$$

\subsection{Expressions for electromagnetic wave components in a three layer waveguide structure (Fig.2, b). Dispersion relations.}

\subsubsection{The case of a $\varepsilon_{1}>\varepsilon_{2}>\varepsilon_{3}$}

For the first (inner) layer $\left(\varepsilon_{1}, \mu_{1}\right)$ :

$$
\left.\begin{array}{c}
H_{z 1}^{m}=A_{1} J_{m}\left(k_{1} r\right) e^{ \pm i m \varphi} \\
E_{z 1}^{e}=B_{1} J_{m}\left(k_{1} r\right) e^{ \pm i m \varphi} \\
H_{\varphi 1}= \pm \frac{\beta m}{r k_{1}^{2}} A_{1} J_{m}\left(k_{1} r\right) e^{ \pm i m \varphi}+\frac{i \omega \varepsilon_{0} \varepsilon_{1}}{k_{1}} B_{1} J_{m}^{\prime}\left(k_{1} r\right) e^{ \pm i m \varphi} \\
E_{\varphi 1}= \pm \frac{\beta m}{r k_{1}^{2}} B_{1} J_{m}\left(k_{1} r\right) e^{ \pm i m \varphi}+\frac{i \omega \mu_{0} \mu_{1}}{k_{1}} A_{1} J_{m}^{\prime}\left(k_{1} r\right) e^{ \pm i m \varphi}
\end{array}\right\}
$$


For the second layer $\left(\varepsilon_{2}, \mu_{2}\right)$ :

$$
\left.\begin{array}{c}
H_{z 2}^{m}=\left[A_{2} I_{m}\left(k_{2} r\right)+D_{2} K_{m}\left(k_{2} r\right)\right] e^{ \pm i m \varphi} \\
E_{z 2}^{e}=\left[B_{2} I_{m}\left(k_{2} r\right)+C_{2} K_{m}\left(k_{2} r\right)\right] e^{ \pm i m \varphi} \\
H_{\varphi 2}= \pm \frac{\beta m}{r k_{2}^{2}}\left[A_{2} I_{m}\left(k_{2} r\right)+D_{2} K_{m}\left(k_{2} r\right)\right] e^{ \pm i m \varphi}+\frac{i \omega \varepsilon_{0} \varepsilon_{2}}{k_{2}}\left[B_{2} I_{m}^{\prime}\left(k_{2} r\right)+C_{2} K_{m}^{\prime}\left(k_{2} r\right)\right] e^{ \pm i m \varphi} \\
E_{\varphi 2}= \pm \frac{\beta m}{r k_{2}^{2}}\left[B_{2} I_{m}\left(k_{2} r\right)+C_{2} K_{m}\left(k_{2} r\right)\right] e^{ \pm i m \varphi}+\frac{i \omega \mu_{0} \mu_{2}}{k_{2}}\left[A_{2} I_{m}^{\prime}\left(k_{2} r\right)+D_{2} K_{m}^{\prime}\left(k_{2} r\right)\right] e^{ \pm i m \varphi}
\end{array}\right\}
$$

For the third (outer) layer $\left(\varepsilon_{3,} \mu_{3}\right)$ :

$$
\left.\begin{array}{c}
H_{z 3}^{m}=A_{3} K_{m}\left(k_{3} r\right) e^{ \pm i m \varphi} \\
E_{z 3}^{e}=B_{3} K_{m}\left(k_{3} r\right) e^{ \pm i m \varphi} \\
H_{\varphi 3}= \pm \frac{\beta m}{r k_{3}^{2}} A_{3} K_{m}\left(k_{3} r\right) e^{ \pm i m \varphi}+\frac{i \omega \varepsilon_{0} \varepsilon_{3}}{k_{3}} B_{3} K_{m}^{\prime}\left(k_{3} r\right) e^{ \pm i m \varphi} \\
E_{\varphi 3}= \pm \frac{\beta m}{r k_{3}^{2}} B_{3} K_{m}\left(k_{3} r\right) e^{ \pm i m \varphi}+\frac{i \omega \mu_{0} \mu_{3}}{k_{3}} A_{3} K_{m}^{\prime}\left(k_{3} r\right) e^{ \pm i m \varphi}
\end{array}\right\}
$$

\subsubsection{The case of a $\varepsilon_{2}>\varepsilon_{1}>\varepsilon_{3}$}

For the first (inner) layer $\left(\varepsilon_{1}, \mu_{1}\right)$ :

$$
\left.\begin{array}{c}
H_{z 1}^{m}=A_{1} I_{m}\left(k_{1} r\right) e^{ \pm i m \varphi} \\
E_{z 1}^{e}=B_{1} I_{m}\left(k_{1} r\right) e^{ \pm i m \varphi} \\
H_{\varphi 1}= \pm \frac{\beta m}{r k_{1}^{2}} A_{1} I_{m}\left(k_{1} r\right) e^{ \pm i m \varphi}+\frac{i \omega \varepsilon_{0} \varepsilon_{1}}{k_{1}} B_{1} I_{m}^{\prime}\left(k_{1} r\right) e^{ \pm i m \varphi} \\
E_{\varphi 1}= \pm \frac{\beta m}{r k_{1}^{2}} B_{1} I_{m}\left(k_{1} r\right) e^{ \pm i m \varphi}+\frac{i \omega \mu_{0} \mu_{1}}{k_{1}} A_{1} I_{m}^{\prime}\left(k_{1} r\right) e^{ \pm i m \varphi}
\end{array}\right\}
$$

For the second (inner) layer $\left(\varepsilon_{2}, \mu_{2}\right)$ :

$$
\left.\begin{array}{c}
H_{z 2}^{m}=\left[A_{2} J_{m}\left(k_{2} r\right)+D_{2} N_{m}\left(k_{2} r\right)\right] e^{ \pm i m \varphi} \\
E_{z 2}^{e}=\left[B_{2} J_{m}\left(k_{2} r\right)+C_{2} N_{m}\left(k_{2} r\right)\right] e^{ \pm i m \varphi} \\
H_{\varphi 2}= \pm \frac{\beta m}{r k_{2}^{2}}\left[A_{2} J_{m}\left(k_{2} r\right)+D_{2} N_{m}\left(k_{2} r\right)\right] e^{ \pm i m \varphi}+\frac{i \omega \varepsilon_{0} \varepsilon_{2}}{k_{2}}\left[B_{2} J_{m}^{\prime}\left(k_{2} r\right)+C_{2} N_{m}^{\prime}\left(k_{2} r\right)\right] e^{ \pm i m \varphi} \\
\left.E_{\varphi 2}= \pm \frac{\beta m}{r k_{2}^{2}}\left[B_{2} J_{m}\left(k_{2} r\right)+C_{2} N_{m}\left(k_{2} r\right)\right]\right]^{ \pm i m \varphi}+\frac{i \omega \mu_{0} \mu_{2}}{k_{2}}\left[A_{2} J_{m}^{\prime}\left(k_{2} r\right)+D_{2} N_{m}^{\prime}\left(k_{2} r\right)\right] e^{ \pm i m \varphi}
\end{array}\right\}
$$

For the third (outer) layer $\left(\varepsilon_{3}, \mu_{3}\right)$ :

$$
\left.\begin{array}{c}
H_{z 3}^{m}=A_{3} K_{m}\left(k_{3} r\right) e^{ \pm i m \varphi} \\
E_{z 3}^{e}=B_{3} K_{m}\left(k_{3} r\right) e^{ \pm i m \varphi} \\
H_{\varphi 3}= \pm \frac{\beta m}{r k_{3}^{2}} A_{3} K_{m}\left(k_{3} r\right) e^{ \pm i m \varphi}+\frac{i \omega \varepsilon_{0} \varepsilon_{3}}{k_{3}} B_{3} K_{m}^{\prime}\left(k_{3} r\right) e^{ \pm i m \varphi} \\
E_{\varphi 3}= \pm \frac{\beta m}{r k_{3}^{2}} B_{3} K_{m}\left(k_{3} r\right) e^{ \pm i m \varphi}+\frac{i \omega \mu_{0} \mu_{3}}{k_{3}} A_{3} K_{m}^{\prime}\left(k_{3} r\right) e^{ \pm i m \varphi}
\end{array}\right\}
$$




\subsubsection{Dispersion relations}

Similarly to the case of a two-layer waveguide structure using the conditions of component continuity at the layer boundaries $\left(r=a: E_{\varphi 1}(a)=E_{\varphi 2}(a), H_{\varphi 1}(a)=H_{\varphi 2}(a), E_{z 1}(a)=E_{z 2}(a)\right.$, $\left.H_{z 1}(a)=H_{z 2}(a) ; \quad r=b: \quad E_{\varphi 2}(b)=E_{\varphi 3}(b), \quad H_{\varphi 2}(b)=H_{\varphi 3}(b), \quad E_{z 2}(b)=E_{z 3}(b), \quad H_{z 2}(b)=H_{z 3}(b)\right) \quad$ the dispersion relation is obtained:

$$
\frac{\frac{\delta_{2}+\delta_{1}\left(\alpha_{2}-\tau_{2} \frac{\alpha_{1}}{\tau_{1}}-\frac{\alpha_{2}}{\alpha_{1}}\right)}{\alpha_{1}\left(\alpha_{2}-\tau_{2} \frac{\alpha_{1}}{\tau_{1}}\right)} \tau_{1}+\frac{\frac{\delta_{1}}{\alpha_{1}} \alpha_{2}-\delta_{2}}{\frac{\tau_{1}}{\alpha_{1}} \alpha_{2}-\tau_{2}} \tau_{3}-\delta_{3}}{\frac{\delta_{2}+\delta_{1}\left(\alpha_{2}-\tau_{2} \frac{\alpha_{1}}{\tau_{1}}-\frac{\alpha_{2}}{\alpha_{1}}\right)}{\alpha_{1}\left(\alpha_{2}-\tau_{2} \frac{\alpha_{1}}{\tau_{1}}\right)} \tau_{2}+\frac{\delta_{1}-\frac{\frac{\xi_{1}}{\alpha_{1}} \alpha_{2}-\xi_{2}}{\frac{\tau_{1}}{\alpha_{1}} \alpha_{2}-\tau_{2}} \tau_{3}-\frac{\xi_{2}+\xi_{1}\left(\alpha_{2}-\tau_{2} \frac{\alpha_{1}}{\tau_{1}}-\frac{\alpha_{2}}{\alpha_{1}}\right)}{\alpha_{1}} \alpha_{2}-\tau_{2}}{\alpha_{4}-\delta_{4}} \tau_{1} \delta_{2}-\frac{\left.\frac{\delta_{1}}{\alpha_{1}} \alpha_{2}-\tau_{2} \frac{\alpha_{1}}{\tau_{1}}\right)}{\frac{\delta_{1}}{\alpha_{1}} \alpha_{2}-\tau_{2}} \tau_{4}-\frac{\xi_{2}+\xi_{1}\left(\alpha_{2}-\tau_{2} \frac{\alpha_{1}}{\tau_{1}}-\frac{\alpha_{2}}{\alpha_{1}}\right)}{\alpha_{1}\left(\alpha_{2}-\tau_{2} \frac{\alpha_{1}}{\tau_{1}}\right)} \tau_{2}}
$$

In the expression (12) the variables are as follows:

$$
\begin{aligned}
& \alpha_{1\left(\varepsilon_{1}>\varepsilon_{2}>\varepsilon_{3}\right)}=\left[\frac{i \omega \mu_{0} \mu_{1}}{k_{1}} \frac{J_{m}^{\prime}\left(k_{1} a\right)}{J_{m}\left(k_{1} a\right)}-\frac{i \omega \mu_{0} \mu_{2}}{k_{2}} \frac{I_{m}^{\prime}\left(k_{2} a\right)}{I_{m}\left(k_{2} a\right)}\right] I_{m}\left(k_{2} a\right) \\
& \alpha_{1\left(\varepsilon_{2}>\varepsilon_{1}>\varepsilon_{3}\right)}=\left[\frac{i \omega \mu_{0} \mu_{1}}{k_{1}} \frac{I_{m}^{\prime}\left(k_{1} a\right)}{I_{m}\left(k_{1} a\right)}-\frac{i \omega \mu_{0} \mu_{2}}{k_{2}} \frac{J_{m}^{\prime}\left(k_{2} a\right)}{J_{m}\left(k_{2} a\right)}\right] J_{m}\left(k_{2} a\right) \\
& \alpha_{2\left(\varepsilon_{1}>\varepsilon_{2}>\varepsilon_{3}\right)}=\left[\frac{i \omega \mu_{0} \mu_{2}}{k_{2}} \frac{I_{m}^{\prime}\left(k_{1} b\right)}{I_{m}\left(k_{1} b\right)}-\frac{i \omega \mu_{0} \mu_{3}}{k_{3}} \frac{K_{m}^{\prime}\left(k_{2} b\right)}{K_{m}\left(k_{2} b\right)}\right] I_{m}\left(k_{2} b\right) \\
& \alpha_{2\left(\varepsilon_{2}>\varepsilon_{1}>\varepsilon_{3}\right)}=\left[\frac{i \omega \mu_{0} \mu_{2}}{k_{2}} \frac{J_{m}^{\prime}\left(k_{1} b\right)}{J_{m}\left(k_{1} b\right)}-\frac{i \omega \mu_{0} \mu_{3}}{k_{3}} \frac{K_{m}^{\prime}\left(k_{3} b\right)}{K_{m}\left(k_{3} b\right)}\right] J_{m}\left(k_{2} b\right) \\
& \tau_{1\left(\varepsilon_{1}>\varepsilon_{2}>\varepsilon_{3}\right)}=\left[ \pm \frac{\beta m}{a k_{1}^{2}} \mp \frac{\beta m}{a k_{2}^{2}}\right] I_{m}\left(k_{2} a\right) \\
& \tau_{1\left(\varepsilon_{2}>\varepsilon_{1}>\varepsilon_{3}\right)}=\left[ \pm \frac{\beta m}{a k_{1}^{2}} \mp \frac{\beta m}{a k_{2}^{2}}\right] J_{m}\left(k_{2} a\right) \\
& \tau_{2\left(\varepsilon_{1}>\varepsilon_{2}>\varepsilon_{3}\right)}=\left[ \pm \frac{\beta m}{b k_{2}^{2}} \mp \frac{\beta m}{b k_{3}^{2}}\right] I_{m}\left(k_{2} b\right) \\
& \tau_{2\left(\varepsilon_{2}>\varepsilon_{1}>\varepsilon_{3}\right)}=\left[ \pm \frac{\beta m}{b k_{2}^{2}} \mp \frac{\beta m}{b k_{3}^{2}}\right] J_{m}\left(k_{2} b\right) \\
& \tau_{3\left(\varepsilon_{1}>\varepsilon_{2}>\varepsilon_{3}\right)}=\left[\frac{i \omega \varepsilon_{0} \varepsilon_{1}}{k_{1}} \frac{J_{m}^{\prime}\left(k_{1} a\right)}{J_{m}\left(k_{1} a\right)}-\frac{i \omega \varepsilon_{0} \varepsilon_{2}}{k_{2}} \frac{I_{m}^{\prime}\left(k_{2} a\right)}{I_{m}\left(k_{2} a\right)}\right] I_{m}\left(k_{2} a\right) \\
& \tau_{3\left(\varepsilon_{2}>\varepsilon_{1}>\varepsilon_{3}\right)}=\left[\frac{i \omega \varepsilon_{0} \varepsilon_{1}}{k_{1}} \frac{I_{m}^{\prime}\left(k_{1} a\right)}{I_{m}\left(k_{1} a\right)}-\frac{i \omega \varepsilon_{0} \varepsilon_{2}}{k_{2}} \frac{J_{m}^{\prime}\left(k_{2} a\right)}{J_{m}\left(k_{2} a\right)}\right] J_{m}\left(k_{2} a\right) \\
& \tau_{4\left(\varepsilon_{1}>\varepsilon_{2}>\varepsilon_{3}\right)}=\left[\frac{i \omega \varepsilon_{0} \varepsilon_{2}}{k_{2}} \frac{I_{m}^{\prime}\left(k_{2} b\right)}{I_{m}\left(k_{2} b\right)}-\frac{i \omega \varepsilon_{0} \varepsilon_{3}}{k_{3}} \frac{K_{m}^{\prime}\left(k_{3} b\right)}{K_{m}\left(k_{3} b\right)}\right] I_{m}\left(k_{2} b\right) \\
& \tau_{4\left(\varepsilon_{2}>\varepsilon_{1}>\varepsilon_{3}\right)}=\left[\frac{i \omega \varepsilon_{0} \varepsilon_{2}}{k_{2}} \frac{J_{m}^{\prime}\left(k_{2} b\right)}{J_{m}\left(k_{2} b\right)}-\frac{i \omega \varepsilon_{0} \varepsilon_{3}}{k_{3}} \frac{K_{m}^{\prime}\left(k_{3} b\right)}{K_{m}\left(k_{3} b\right)}\right] J_{m}\left(k_{2} b\right)
\end{aligned}
$$




$$
\begin{aligned}
& \delta_{1\left(\varepsilon_{1}>\varepsilon_{2}>\varepsilon_{3}\right)}=\left[ \pm \frac{\beta m}{a k_{2}^{2}} \mp \frac{\beta m}{a k_{1}^{2}}\right] K_{m}\left(k_{2} a\right) \\
& \delta_{1\left(\varepsilon_{2}>\varepsilon_{1}>\varepsilon_{3}\right)}=\left[ \pm \frac{\beta m}{a k_{2}^{2}} \mp \frac{\beta m}{a k_{1}^{2}}\right] N_{m}\left(k_{2} a\right) \\
& \delta_{2\left(\varepsilon_{1}>\varepsilon_{2}>\varepsilon_{3}\right)}=\left[ \pm \frac{\beta m}{b k_{3}^{2}} \mp \frac{\beta m}{b k_{2}^{2}}\right] N_{m}\left(k_{2} b\right) \\
& \delta_{2\left(\varepsilon_{2}>\varepsilon_{1}>\varepsilon_{3}\right)}=\left[ \pm \frac{\beta m}{b k_{3}^{2}} \mp \frac{\beta m}{b k_{2}^{2}}\right] N_{m}\left(k_{2} b\right) \\
& \delta_{3\left(\varepsilon_{1}>\varepsilon_{2}>\varepsilon_{3}\right)}=\left[\frac{i \omega \varepsilon_{0} \varepsilon_{2}}{k_{2}} \frac{K_{m}^{\prime}\left(k_{2} a\right)}{K_{m}\left(k_{2} a\right)}-\frac{i \omega \varepsilon_{0} \varepsilon_{1}}{k_{1}} \frac{J_{m}^{\prime}\left(k_{1} a\right)}{J_{m}\left(k_{1} a\right)}\right] K_{m}\left(k_{2} a\right) \\
& \delta_{3\left(\varepsilon_{2}>\varepsilon_{1}>\varepsilon_{3}\right)}=\left[\frac{i \omega \varepsilon_{0} \varepsilon_{2}}{k_{2}} \frac{N_{m}^{\prime}\left(k_{2} a\right)}{N_{m}\left(k_{2} a\right)}-\frac{i \omega \varepsilon_{0} \varepsilon_{1}}{k_{1}} \frac{I_{m}^{\prime}\left(k_{1} a\right)}{I_{m}\left(k_{1} a\right)}\right] N_{m}\left(k_{2} a\right) \\
& \delta_{4\left(\varepsilon_{1}>\varepsilon_{2}>\varepsilon_{3}\right)}=\left[\frac{i \omega \varepsilon_{0} \varepsilon_{3}}{k_{3}} \frac{K_{m}^{\prime}\left(k_{3} b\right)}{K_{m}\left(k_{3} b\right)}-\frac{i \omega \varepsilon_{0} \varepsilon_{2}}{k_{2}} \frac{K_{m}^{\prime}\left(k_{2} b\right)}{K_{m}\left(k_{2} b\right)}\right] K_{m}\left(k_{2} b\right) \\
& \delta_{4\left(\varepsilon_{2}>\varepsilon_{1}>\varepsilon_{3}\right)}=\left[\frac{i \omega \varepsilon_{0} \varepsilon_{3}}{k_{3}} \frac{K_{m}^{\prime}\left(k_{3} b\right)}{K_{m}\left(k_{3} b\right)}-\frac{i \omega \varepsilon_{0} \varepsilon_{2}}{k_{2}} \frac{N_{m}^{\prime}\left(k_{2} b\right)}{N_{m}\left(k_{2} b\right)}\right] N_{m}\left(k_{2} b\right) \\
& \xi_{1\left(\varepsilon_{1}>\varepsilon_{2}>\varepsilon_{3}\right)}=\left[\frac{i \omega \mu_{0} \mu_{2}}{k_{2}} \frac{K_{m}^{\prime}\left(k_{2} a\right)}{K_{m}\left(k_{2} a\right)}-\frac{i \omega \mu_{0} \mu_{1}}{k_{1}} \frac{J_{m}^{\prime}\left(k_{1} a\right)}{J_{m}\left(k_{1} a\right)}\right] K_{m}\left(k_{2} a\right) \\
& \xi_{1\left(\varepsilon_{2}>\varepsilon_{1}>\varepsilon_{3}\right)}=\left[\frac{i \omega \mu_{0} \mu_{2}}{k_{2}} \frac{N_{m}^{\prime}\left(k_{2} a\right)}{N_{m}\left(k_{2} a\right)}-\frac{i \omega \mu_{0} \mu_{1}}{k_{1}} \frac{I_{m}^{\prime}\left(k_{1} a\right)}{I_{m}\left(k_{1} a\right)}\right] N_{m}\left(k_{2} a\right) \\
& \xi_{2\left(\varepsilon_{1}>\varepsilon_{2}>\varepsilon_{3}\right)}=\left[\frac{i \omega \mu_{0} \mu_{3}}{k_{3}} \frac{K_{m}^{\prime}\left(k_{3} b\right)}{K_{m}\left(k_{3} b\right)}-\frac{i \omega \mu_{0} \mu_{2}}{k_{2}} \frac{K_{m}^{\prime}\left(k_{2} b\right)}{K_{m}\left(k_{2} b\right)}\right] K_{m}\left(k_{2} b\right) \\
& \xi_{2\left(\varepsilon_{2}>\varepsilon_{1}>\varepsilon_{3}\right)}=\left[\frac{i \omega \mu_{0} \mu_{3}}{k_{3}} \frac{K_{m}^{\prime}\left(k_{3} b\right)}{K_{m}\left(k_{3} b\right)}-\frac{i \omega \mu_{0} \mu_{2}}{k_{2}} \frac{N_{m}^{\prime}\left(k_{2} b\right)}{N_{m}\left(k_{2} b\right)}\right] N_{m}\left(k_{2} b\right)
\end{aligned}
$$

\section{Power Flow Density}

The longitudinal component of the Pointing vector determines the average energy density along the waveguide. The expression for the total power flow through the section of the waveguide:

$$
P=\frac{1}{2} \operatorname{Re} \int_{S c r .}[\vec{E} \times \vec{H}] \vec{e}_{z} d S=\frac{1}{2} \int_{0}^{2 \pi} \int_{0}^{R} \operatorname{Re}\left(E_{r} H_{\varphi}-E_{\varphi} H_{r}\right) r d r d \varphi
$$

4.1 The case of a three-layer waveguide structure $\left(\varepsilon_{1}>\varepsilon_{2}>\varepsilon_{3}\right)$ in the absence of dependence on the azimuth angle $(m=0)$ of the transverse magnetic (TM) wave type

Particular cases of power flux density through the cross-section of the waveguide in individual areas: 


$$
\left.\begin{array}{c}
P_{1}=A^{2} \frac{\beta \omega \varepsilon_{1} a^{2}}{8 k_{1}^{2}}\left[J_{1}^{2}\left(k_{1} a\right)-J_{0}\left(k_{1} a\right) J_{2}\left(k_{1} a\right)\right], \\
P_{2}=B^{2} \frac{\beta \omega \varepsilon_{2}}{8 k_{2}^{2}} r^{2}\left[I_{1}^{2}\left(k_{2} r\right)-\left.I_{0}\left(k_{2} r\right) I_{2}\left(k_{2} r\right)\right|_{a} ^{b}-B C \frac{\beta \omega \varepsilon_{2}}{2 k_{2}^{2}} \int_{a}^{b} I_{1}\left(k_{2} r\right) K_{1}\left(k_{2} r\right) r d r+\right. \\
+\left.C^{2} \frac{\beta \omega \varepsilon_{2}}{8 k_{2}^{2}} r^{2}\left[K_{1}^{2}\left(k_{2} r\right)-K_{0}\left(k_{2} r\right) K_{2}\left(k_{2} r\right)\right]\right|_{a} ^{b}, \\
P_{3}=D^{2} \frac{\beta \omega \varepsilon_{3} b^{2}}{8 k_{3}^{2}}\left[K_{0}\left(k_{3} b\right) K_{2}\left(k_{3} b\right)-K_{1}^{2}\left(k_{3} b\right)\right]
\end{array}\right\}
$$

In the second region (22) the integrals are solved by numerical and approximate methods.

4.2 The case of a three-layer waveguide structure $\left(\varepsilon_{2}>\varepsilon_{1}>\varepsilon_{3}\right)$ in the absence of dependence on the azimuth angle $(m=0)$ of the transverse magnetic (TM) wave type

$$
\left.\begin{array}{c}
P_{1}=A^{2} \frac{\beta \omega \varepsilon_{1} a^{2}}{8 k_{1}^{2}}\left[I_{1}^{2}\left(k_{1} a\right)-I_{0}\left(k_{1} a\right) I_{2}\left(k_{1} a\right)\right], \\
P_{2}=B^{2} \frac{\beta \omega \varepsilon_{2}}{8 k_{2}^{2}} r^{2}\left[J_{1}^{2}\left(k_{2} r\right)-\left.J_{0}\left(k_{2} r\right) J_{2}\left(k_{2} r\right)\right|_{a} ^{b}+B C \frac{\beta \omega \varepsilon_{2}}{2 k_{2}^{2}} \int_{a}^{b} J_{1}\left(k_{2} r\right) N_{1}\left(k_{2} r\right) r d r+\right. \\
+C^{2} \frac{\beta \omega \varepsilon_{2}}{8 k_{2}^{2}} r^{2}\left[N_{1}^{2}\left(k_{2} r\right)-\left.N_{0}\left(k_{2} r\right) N_{2}\left(k_{2} r\right)\right|_{a} ^{b},\right. \\
P_{3}=D^{2} \frac{\beta \omega \varepsilon_{3} b^{2}}{8 k_{3}^{2}}\left[K_{0}\left(k_{3} b\right) K_{2}\left(k_{3} b\right)-K_{1}^{2}\left(k_{3} b\right)\right]
\end{array}\right\}
$$

4.3 The cases of a two-layer waveguide structure $\left(r \leq a, \varepsilon_{1} ; r \geq a, \varepsilon_{2} ; \varepsilon_{1}>\varepsilon_{2}\right)$ in the absence of dependence on the azimuth angle $(m=0)$ of the transverse magnetic (TM) and transverse electric (TE) wave types

$$
\left.\begin{array}{c}
P_{1 T M}=\frac{\beta \omega \varepsilon_{1}}{8 \pi k_{1}^{2}} \operatorname{Re} \int_{0}^{2 \pi a} \int_{0} J_{1}^{2}\left(k_{1} r\right) r d r d \varphi, \\
P_{2 T M}=\frac{\beta \omega \varepsilon_{2}}{8 \pi k_{2}^{2}}\left[\frac{J_{0}\left(k_{1} a\right)}{K_{0}\left(k_{2} a\right)}\right]^{2} \operatorname{Re} \int_{0}^{2 \pi \infty} \int_{a}^{\infty} K_{1}^{2}\left(k_{2} r\right) r d r d \varphi . \\
P_{1 T E}=\frac{\beta \omega \mu_{1}}{8 \pi k_{1}^{2}} \frac{1}{2} \operatorname{Re} \int_{0}^{2 \pi a} \int_{0}^{2} J_{1}^{2}\left(k_{1} r\right) r d r d \varphi, \\
P_{2 T E}=\frac{\beta \omega \mu_{2}}{8 \pi k_{2}^{2}}\left[\frac{J_{0}\left(k_{1} a\right)}{K_{0}\left(k_{2} a\right)}\right]^{2} \operatorname{Re} \int_{0}^{2 \pi \infty} \int_{a}^{\infty} K_{1}^{2}\left(k_{2} r\right) r d r d \varphi .
\end{array}\right\}
$$

\section{Calculation results}

Calculations were made on frequencies $\omega=5,5^{*} 10^{11} . .10^{*} 10^{11}$ (i.e. $v \sim 10^{2} \mathrm{GHz}$ ). 
For the three-layer waveguide system, dispersion relations for the case of $\varepsilon_{2}>\varepsilon_{1}>\varepsilon_{3}$ for TE- and TM-waves were obtained:

$$
\begin{aligned}
& \frac{J_{0}\left(k_{2} a\right)}{J_{0}\left(k_{2} b\right)}\left[\frac{1}{k_{1}} \bar{\mu} \frac{I_{1}\left(k_{1} a\right)}{I_{0}\left(k_{1} a\right)}+\frac{1}{k_{2}} \frac{J_{1}\left(k_{2} a\right)}{J_{0}\left(k_{2} a\right)}\right]\left[\frac{1}{k_{2}} \frac{N_{1}\left(k_{2} b\right)}{N_{0}\left(k_{2} b\right)}-\frac{1}{k_{3}} \overline{\bar{\mu}} \frac{K_{1}\left(k_{3} b\right)}{K_{0}\left(k_{3} b\right)}\right]- \\
& -\left[\frac{1}{k_{2}} \frac{J_{1}\left(k_{2} b\right)}{J_{0}\left(k_{2} b\right)}-\frac{1}{k_{3}} \overline{\bar{\mu}} \frac{K_{1}\left(k_{3} b\right)}{K_{0}\left(k_{3} b\right)}\right]\left[\frac{1}{k_{1}} \bar{\mu} \frac{I_{1}\left(k_{1} a\right)}{I_{0}\left(k_{1} a\right)}+\frac{1}{k_{2}} \frac{N_{1}\left(k_{2} a\right)}{N_{0}\left(k_{2} a\right)}\right] \frac{N_{0}\left(k_{2} a\right)}{N_{0}\left(k_{2} b\right)}=0 \\
& \frac{J_{0}\left(k_{2} a\right)}{J_{0}\left(k_{2} b\right)}\left[\frac{1}{k_{1}} \bar{\varepsilon} \frac{I_{1}\left(k_{1} a\right)}{I_{0}\left(k_{1} a\right)}-\frac{1}{k_{2}} \frac{J_{1}\left(k_{2} a\right)}{J_{0}\left(k_{2} a\right)}\right]\left[\frac{1}{k_{2}} \frac{N_{1}\left(k_{2} b\right)}{N_{0}\left(k_{2} b\right)}+\frac{1}{k_{3}} \overline{\bar{\varepsilon}} \frac{K_{1}\left(k_{3} b\right)}{K_{0}\left(k_{3} b\right)}\right]- \\
& -\left[\frac{1}{k_{2}} \frac{J_{1}\left(k_{2} b\right)}{J_{0}\left(k_{2} b\right)}+\frac{1}{k_{3}} \overline{\bar{\varepsilon}} \frac{K_{1}\left(k_{3} b\right)}{K_{0}\left(k_{3} b\right)}\right]\left[\frac{1}{k_{1}} \bar{\varepsilon} \frac{I_{1}\left(k_{1} a\right)}{I_{0}\left(k_{1} a\right)}-\frac{1}{k_{2}} \frac{N_{1}\left(k_{2} a\right)}{N_{0}\left(k_{2} a\right)}\right] \frac{N_{0}\left(k_{2} a\right)}{N_{0}\left(k_{2} b\right)}=0
\end{aligned}
$$

In these expressions the variables are as follows $\bar{\mu}=\frac{\mu_{1}}{\mu_{2}}, \overline{\bar{\mu}}=\frac{\mu_{3}}{\mu_{2}}, \bar{\varepsilon}=\frac{\varepsilon_{1}}{\varepsilon_{2}}, \overline{\bar{\varepsilon}}=\frac{\varepsilon_{3}}{\varepsilon_{2}}, k_{n}$ transverse wave numbers in separate layers.

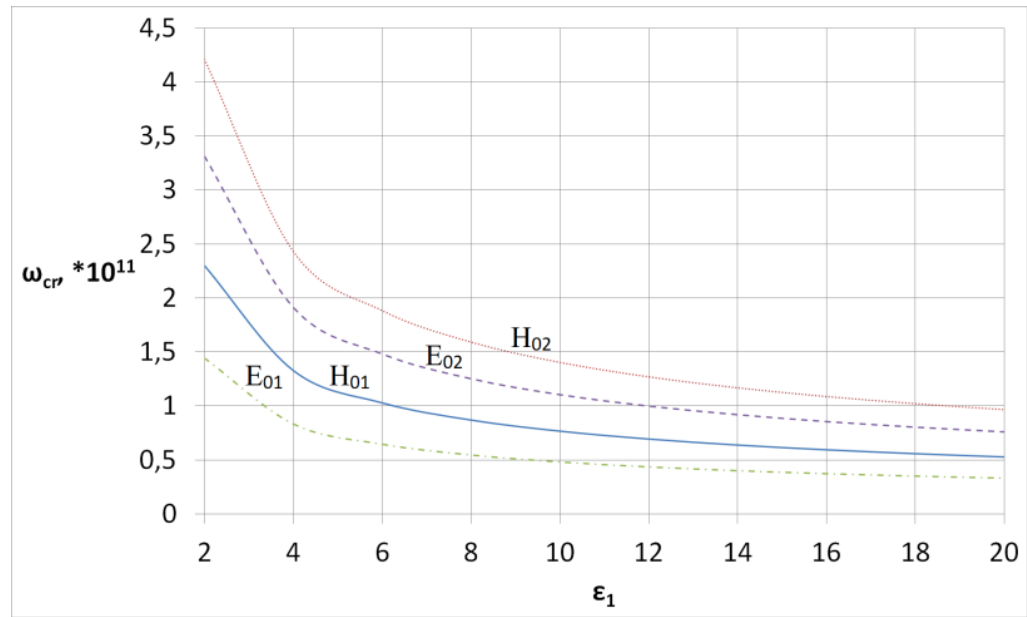

Fig. 3. Dependences $\omega_{\mathrm{cr}}\left(\varepsilon_{1}\right)$ of critical frequency on the relative dielectric permittivity of the inner layer of the waveguide at a fixed value of $\varepsilon_{2}=1$ (dielectric permittivity of the outer layer) and the following parameters: $\mu_{1}=1, \mu_{2}=1, a=0,005 \mathrm{~m}$.

The characteristics of magnetic waves in the two-layer waveguide structure are analyzed, the curves corresponding to the dispersion equation for the case $m=0$ are constructed to determine the frequencies with which the waves can propagate in the waveguide of a given configuration, the critical frequency is calculated. Graphs of the critical frequency dependence on dielectric permittivity for individual modes of waves are obtained (Fig. 3). It can be seen that with the growth of the relative dielectric permittivity the value of the critical frequency decreases.

According to the results of the calculation, the plots of the relative power flow density $\mathrm{p} / \mathrm{p}^{*}$ (Fig. 4) through the cross-section of the waveguide on the value of $\mathrm{r} / \mathrm{a}$ for the case of TM waves in a two-layer waveguide structure at $\varepsilon_{2}=2$ and different values of $\varepsilon_{1}$ are plotted. The curves of the relative power flux density $\left(\mathrm{p} / \mathrm{p}^{*}\right)$ of TM waves in the three-layer waveguide structure (Fig.5) on the value of r/a for fixed $\varepsilon_{2}=5, \varepsilon_{3}=1$ and different values of dielectric permittivity $\varepsilon_{1}=2 ; 3,8 ; 6,5 ; 7 ; 10$ are also plotted. 


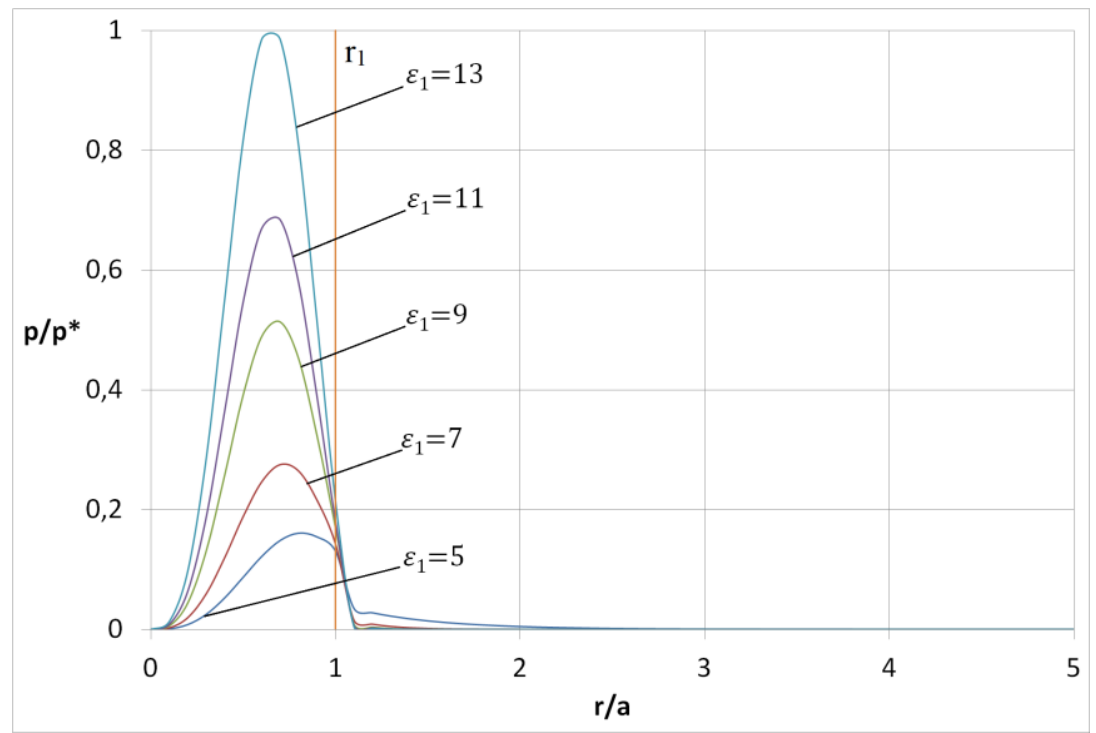

Fig. 4. Dependence of the relative power $\left(\mathrm{p} / \mathrm{p}^{*}\right)$ on the value of $\mathrm{r} / \mathrm{a}$ (where a is the cross section radius of the first layer) for/at a fixed value of $\varepsilon_{2}=2$ and different values of dielectric constant $\varepsilon_{1}=5 ; 7 ; 9 ; 11 ; 13$. The point $\mathrm{r} / \mathrm{a}=1$ corresponds to the boundary of the internal layer (i.e. $\mathrm{r}_{1}=0,001 \mathrm{~m}$ ).

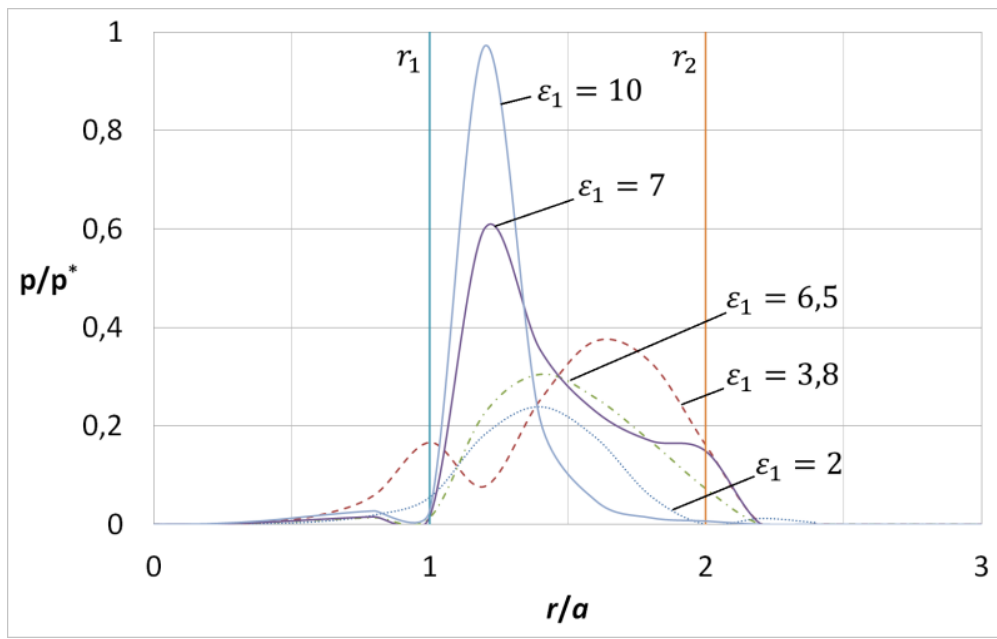

Fig. 5. Dependence of the relative power $\left(\mathrm{p} / \mathrm{p}^{*}\right)$ on the $\mathrm{r} / \mathrm{a}$ for fixed values of $\varepsilon_{2}=5, \varepsilon_{3}=1$ and different values of dielectric constant $\varepsilon_{1}=2 ; 3,8 ; 6,5 ; 7 ; 10$. The points of $\mathrm{r} / \mathrm{a}=1$ and $\mathrm{r} / \mathrm{a}=2$ correspond to the boundaries of two internal layers (i.e. $r_{1}=0,001 \mathrm{~m} ; r_{2}=0,002 \mathrm{~m}$ ).

\section{Conclusions}

Dispersion equations for waves propagating in cylindrical dielectric waveguides are obtained. As the dielectric permittivity of the waveguide increases, the critical frequency decreases, so the cutoff area shifts to the lower frequency range. Thus, by changing the relative dielectric permittivity of the waveguide material, it is possible to achieve the desired frequency range of electromagnetic waves.

According to the graphs on Fig. 4 it can be seen that the greater the value of the dielectric permittivity of the first layer, the higher the concentration of power in the given 
layer. The peak power slightly shifts to the center of the first (inner) layer with the growth of the value of $\varepsilon_{1}$.

According to the graph of the dependence of the power for the electromagnetic waves (TM) on the radial coordinate, it can be seen that at fixed values of the dielectric permittivity of two layers $\left(\varepsilon_{2}=5, \varepsilon_{3}=1\right)$, and the change in the value of $\varepsilon_{1}$ of the internal layer the localization of the main energy changes. As it can be seen, the main power is concentrated in the middle layer, and when the peak power shifts from $r_{2}$ to $r_{1}$, the wave is replaced to the boundary between the first and second layers.

\section{References}

1. Plonis, D., Bučinskas, J. Pomarnacki, R., Miniotas, D.; Paulikas, Š.; Katkevičius, A.; Martavičius, R.; Nickelson, L., Electric Field and Dispersion Characteristic Calculations of Glass Tube Waveguides Filled with Biological Substances, Electronics, 8, 301 (2019)

2. C. Yeh and F. I. Shimabukuro, The essence of dielectric waveguides. (Springer, 2008)

3. Veselov, G.I., Raevskiy S.B., Layered metallodielectric waveguides (Radio and communication, Moscow, 1988)

4. Vziatyshev V. F., Dielectric waveguides (Soviet Radio, Moscow, 1970)

5. D.V. Valovik, E.Yu. Smolkin, Numerical solution of the problem of electromagnetic TM-wave propagation in a round dielectric waveguide filled with nonlinear medium, Izvestia of higher educational institutions, Physical and mathematical sciences, v.3 (23), pp.29-37 (2012)

6. A.D. Grigoriev, Electrodynamics and microwave technology: textbook for higher education institutions on specialty "Electronic devices and devices" (Higher school, Moscow, 1990)

7. J. Jackson, Classical electrodynamics (Russian translation of G.V. Voskresensky and L.S. Solovyov) ("Mir", 1965)

8. I.V. Lebedev, Technics and microwave devices ("Higher School", Moscow, 1970)

9. N.A. Semyonov, Technical electrodynamics ("Communication", Moscow, 1973)

10. Yu. Mitelman, S. Shabunin, Electrodynamics of multilayer cylindrical guiding systems (LAP LAMBERT Academic Publishing, 2013)

11. N.N. Fedorov, Fundamentals of Electrodynamics: A Manual for HEIs ("Higher School", Moscow, 1980) 